\title{
Ultrasonic Measurements of Molecular Relaxation in Ethane and Carbon Monoxide
}

\author{
Pär-Erik Martinsson \& Jerker Delsing \\ EISLAB, Dept. of Computer Science and Electrical Engineering, Luleå University of Technology, \\ SE-971 87 Luleå, SWEDEN \\ e-mail : par-erik@sm.luth.se,jerker.delsing@sm.luth.se
}

\begin{abstract}
This paper describes how molecular relaxation can be measured using ultrasound. The velocity and absorption of sound varies with frequency due to molecular relaxation. By measuring these variation the relaxation strength and the effective relaxation time for singular relaxations can be calculated. This paper describes initial measurements performed in order to survey the sound properties in gases both experimentally and theoretically.
\end{abstract}

\section{INTRODUCTION}

Energy gases, such as natural- and biogas, distributed in the large European and Swedish gas grids account for very high monetary values. With the use of different sources of gas (i.e. different gas fields) the energy content of the gas delivered to customers can vary considerably. Variations up to $20 \%$ are not uncommon and can be on a time scale of seconds [1]. For both customers and gas companies it therefore becomes of importance to measure the energy content to get fair invoice. The two dominating methods to measure energy content is by calorimetry [2] and on-line gas chromatography [3]. Both methods are reliable but they are slow, measurement periods of 15-20 minutes are typical. Today, no method or instrument exists that is both cheap and reliable to meet these needs.

A sonic method, based on measurements of the spectral attenuation and speed of sound in gas mixtures could be a competitor to the above stated measurement methods. This is possible since both the attenuation and velocity of sound is dependent of the gas composition. However, accurate and precise knowledge about each component of any gas mixture is required as a function of temperature and more important as a function of frequency.

The aim of the work presented in this paper was to investigate the acoustical properties of Ethane and Carbon Monoxide. Ethene is an important constituent of natural- and bio gas. Of specially interest was to find the relaxation times associated with Ethane and Carbon Monoxide since no values of them is reported in the literature (to the authors knowledge).

\section{THEORY}

In this section a short introduction to the theory of sound propagation and absorption is given. The difference between classical predictions of the speed of sound and absorption and the theory where molecular relaxation is included is outlined. Finally a description of how molecular relaxation phenomena can be used in a sonic gas energy sensor application is given.

The classical speed of sound, $c_{0}$, is given by [4]

$$
c_{0}^{2}=\frac{R T}{M} \gamma
$$

where $R, T$, and $M$ is the universal gas constant, temperature and molar mass of the gas, respectively. $\gamma$ is the ratio of specific heat at constant pressure $C_{P}$ divided by the specific heat at constant volume $C_{V}$. They are related to each other by the relation $C_{P}-C_{V}=R$. Classical attenuation is caused by friction and heat conduction [4] and is given by

$$
\alpha_{c}=\frac{\omega^{2}}{2 \rho_{0} c^{3}}\left(\frac{4}{3} \eta+(\gamma-1) \frac{\kappa}{C_{p}}\right),
$$

where $\eta$ is the viscosity, $\kappa$ is the thermal conductivity of the fluid and $\omega$ is the frequency in $\mathrm{rad} / \mathrm{s}$.

When the energy of a gas volume is suddenly increased there is a redistribution of energy among the translational degrees of freedom i.e. an increase in pressure. Additionally, a redistribution among the energy states, vibrational and/or rotational degrees of freedom, within the gas molecules occurs. The molecules tend to restore to equilibrium, which usually is referred to as thermal molecular relaxation. If a sound wave is the cause of a sudden energy change of a gas volume this relaxation gives rise to an additional adsorption of sound energy.

Each relaxation is associated with a characteristic time constant, the relaxation time, $\tau$, and the absorption of sound attains a maximum value when the period of the sound wave is in the same order of magnitude as the relaxation time.

Now, assume that one internal state (rotation or vibration) interacts with the external or translational degree of freedom during the passage of a sound wave. Furthermore assume that the transition of energy is associated with a relaxation time, $\tau$. Then the effective value of the molar specific heat at constant volume is given by[2]

$$
\left(C_{v}\right)_{e f f}=C_{e x t}+C_{i n t}(1+i \omega \tau)^{-1}
$$

where $C_{\text {ext }}$ is the specific heat of the external degrees of freedom and $C_{i n t}$ is the specific heat of the internal degree of freedom interacting in the process. We have as $\omega \rightarrow 0$ that $\left(C_{V}\right)_{e f f} \rightarrow C_{e x t}+C_{i n t}=C_{V}^{O}$ and as $\omega \rightarrow \infty$ that $\left(C_{V}\right)_{e f f} \rightarrow$ $C_{\text {ext }}=C_{V}^{\infty}$. It is obvious from (3) and (1) that the speed of sound varies not only with temperature but also with frequency.

The influence of the frequency dependency of the specific heat, on the attenuation, $\alpha$, and speed of sound, $c$, have been investigated elsewhere [8] and is given by

$$
\alpha=\pi r \frac{1+\omega \widetilde{\tau}}{1+\omega^{2} \widetilde{\tau}^{2}(1-r)}
$$

and

$$
\frac{c^{2}}{c_{0}^{2}}=\frac{1+\omega^{2} \widetilde{\tau}^{2}}{1+\omega^{2} \widetilde{\tau}^{2}(1-r)}
$$


where $c_{0}$ is given by (1). The constant $r$ is known as the relaxation strength and is calculated from values of the specific heat in the low and high frequency limits respectively

$$
r=\frac{C_{i n t} R}{C_{P}^{\infty} C_{V}^{0}}=1-\frac{C_{P}^{0} C_{V}^{\infty}}{C_{P}^{\infty} C_{V}^{0}}=1-\frac{c_{\infty}^{2}}{c_{0}^{2}},
$$

where the sub- and superscript notation $\infty$ refers to high frequency and 0 refers to low frequency. The effective relaxation time $\widetilde{\tau}$ is given by

$$
\widetilde{\tau}=\frac{C_{P}^{\infty}}{C_{P}^{0}} \tau .
$$

Energy interactions as described above can only occur during collisions between molecules. Since the collision time (the average time it takes for a collision between two molecules to occur) is proportional to the density [9] so is the time constant, $\tau$. Hence, $\tau$ is inversely proportional to the pressure, in fact $\tau P=$ const. for a perfect gas. The property $\omega \tau$ that appears in (3), (4) and (5) can therefore be increased either by increasing the frequency or by decreasing the pressure.

Typical variation of attenuation and speed of sound for an arbitrary gas with a single relaxation phenomena is given in Fig. (1), for which a value of $\widetilde{\tau} / r=0.1$ was used. For low frequen-

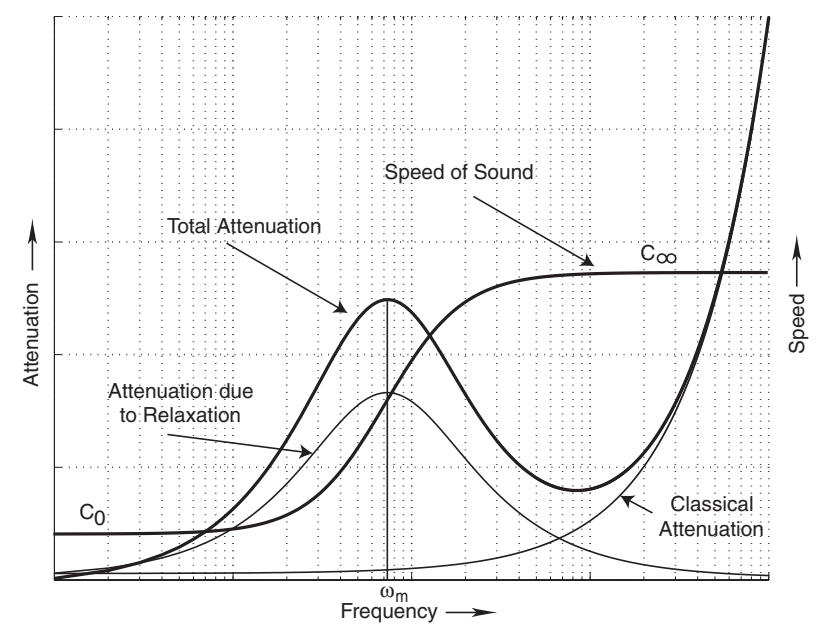

Fig. 1. Typical behavior of the attenuation and speed of sound in a gas with one dominating relaxation phenomena, in the figure the ratio $\widetilde{\tau} / r=0.1$. Notice how the speed attains a constant value for low and high frequencies and the absorbtion peak occurring between these values.

cies the speed of sound is fairly constant and the same is true for high frequencies. Both the classical absorption and the absorption due to molecular relaxation have been drawn out. The total absorption is the sum of these two contributors. Notice how the total attenuation reaches the value of the classical attenuation at high frequencies. The attenuation at any frequency due to molecular relaxation is, however, never less than expected from classical attenuation. Therefore a measurement of absorption either agrees well with classical calculations (at high frequencies) or is larger than predicted from (2).

The relaxation peak occur at a frequency,

$$
\omega_{m}=\frac{1}{\widetilde{\tau} \sqrt{1-r}}=\frac{1}{\tau} \sqrt{\frac{C_{V}^{0} C_{P}^{0}}{C_{V}^{\infty} C_{P}^{\infty}}}
$$

at which the speed of sound attains a value of

$$
c^{2}\left(\omega_{m}\right)=\frac{1}{2}\left(c_{0}^{2}+c_{\infty}^{2}\right)
$$

From an experimental measurement of the attenuation and speed of sound all sufficient parameters needed to describe a gas can be calculated. By using the value the speed of sound reaches for high frequencies, $c_{\infty}$, and the value at low frequencies, $c_{0}$, in (6), the relaxation strength, $r$, can be calculated from experimental data. By identification of the frequency, $\omega_{n}$, where the relaxation peak occurs the effective relaxation time, $\widetilde{\tau}$, can be calculated. This can be done either by examining the attenuation curve, or by using (9) and the velocity curve.

In a mixture of several gases several relaxation phenomena occur. It is possible to extract information about the concentration [5] [6] of each component by study the relaxation spectrum (velocity and absorption) on the frequency to pressure scale. If the gases in a gas mixture can be considered independent of each others i.e. they only exchange energy between molecules of the same kind, it is particularly simple since each peak in a spectrum belongs to a certain gas component. However, several molecular interactions must probably be taken into consideration in most cases [7].

The theory described above is very short but should give the reader a good overview of how the sound properties vary with frequency and pressure for gases with a singular relaxation.

\section{EXPERIMENTS}

A pulse echo measurement scheme was used to measure the attenuation and speed of sound for the gases. The difference in time of flight of the second echo to the first echo gives the speed of sound. The absorption of sound can be calculated by comparing the amplitudes of the first and second echo. The first part of this section describes how the experiments was performed and the instrumentation used. The second part explains how the data was analyzed.

\section{A. Experimental Setup}

The acoustical properties of interest varies with frequency and pressure. There are two ways to achieve a change on that scale for a pulse-echo system. Either transducers of different center frequencies can be used for a fixed pressure or one transducer is used while the pressure is changed. The later of the two principles was chosen in the presented work. A $1 \mathrm{MHz}$ air transducer was used whereas the static pressure of the gas was varied.

In order to achieve different static pressures a special chamber was built, se Fig. (2). The Pressure in the chamber is limited by the maximum pressure delivered by the gas regulators used on the gas bottles. The regulators used gave an output pressure from 0bar to 8bar or 12bar, above atmospheric zero.

All gases described in this paper are highly flammable except for Carbon Monoxide which is also extremely toxic. Due to safety reasons all measurement was performed inside of a fume hood.

The pressure in the chamber was measured with an ANDERSON TPP Pressure Transmitter with a range of up to 13.6bar above atmospheric zero. The transmitter have an accuracy of 




Fig. 2. The measurement equipment. All measurements was performed in a specially constructed pressure chamber.

about $30 \mathrm{mbar}$, this includes the combined effects of linearity, hysteresis and repeatability.

The transducer was mounted on a stainless steel measurement cell, see lower left corner of Fig. (2). The distance between the transducer surface and the reflecting bottom of the measurement cell was measured with a caliper to $71.69 \pm 0.06 \mathrm{~mm}$. The measurement cell was then emerged into the pressure chamber.

To excite and receive acoustic pulses with and from the transducer a Panametrics Pulser/Receiver Model 5072 was used. For the transmitting mode the pulser/receiver was set to deliver maximum energy to the transducer, which corresponds to a short voltage pulse of amplitude $380 \mathrm{~V}$ and of energy $104 \mu \mathrm{J}$. In receiving mode a built in amplifier permits the signals to be amplified up to $59 d B$. For the experiment described in this paper the gain was typically set to $20 d B$ throughout the measurements, but for some of the pulses recorded at low pressure, a higher gain was used. The output of the amplifier is linear up to $\pm 1 \mathrm{~V}$.

All pulses were sampled with a Tektronix TDS 724 1Gs/s Oscilloscope. The attenuation in most gases is very high, hence the amplitude of the second echo is considerably lower than that of the first echo. In order to maximize the bit resolution of the sampled pulses the first and second echo were recorded separately with the vertical scale $(\mathrm{V} / \mathrm{div})$ of the oscilloscope set differently. The delay time between the first and second sampling windows was noted for each pulse. This procedure was possible since the oscilloscope does not effect the phase of the signals for different amplification. It was also confirmed with a measurement. The pulses was sampled 100 times and averaged with the oscilloscope and then saved on a PC. All transfer of data
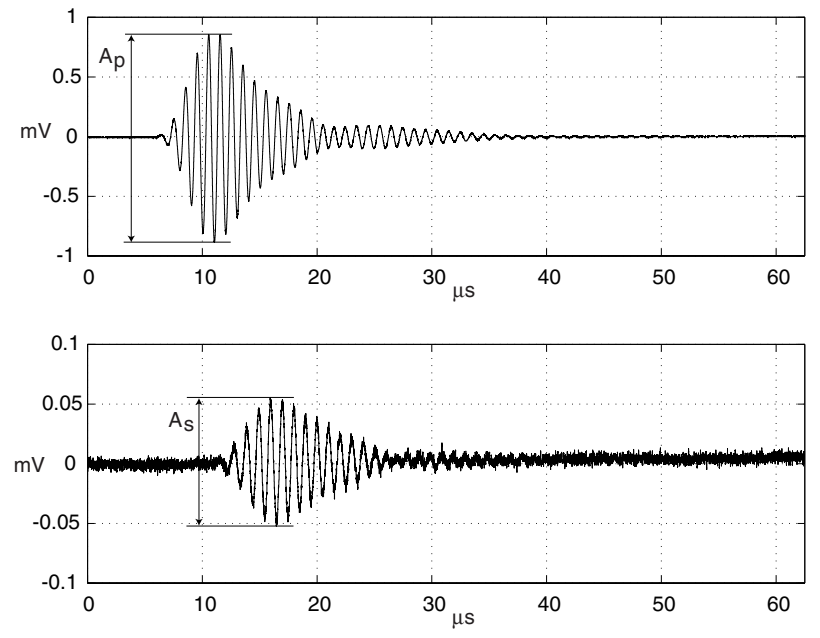

Fig. 3. Primary and secondary echoes measured at 1.8bar in Ethane

was performed trough a GPIB interface between the PC and the oscilloscope.

Temperature measurements were done with an encapsulated PT100 sensor mounted trough the wall of the pressure chamber.

The transducer used was originally designed for operation in air which have an acoustical impedance $Z_{\text {air }}=415$ Pas $/ \mathrm{m}$ ( $\left.T=293^{\circ} C, P=1 \mathrm{bar}\right)$. Since the acoustical impedance of the gases used are different from air the transducer will not operate at its optimal performance, and consequently not transmit as much power as desirable.

The pressure was randomly varied in order do avoid repetitive errors. No leakage of the pressure chamber could be recorded even though it was left for several days containing a high pressure.

\section{B. Data Analysis}

All pulses was sampled with 250000 points during a sampling time of $62.5 \mu \mathrm{s}$. A plot of a typically measurement is given in Fig. (3). The signal to noise floor of the secondary echoes was typically $25-30 d B$ at low pressure. At high pressure the signal to noise floor was typically $50-60 d B$ for both echoes.

The amplitude of a plane wave that passes through the measurement cell decreases due to attenuation as $A=A_{0} e^{-\alpha 2 d}$ where $\alpha$ is the attenuation and $d$ is the distance between the transducer surface and the reflective bottom of the measurement cell. If we assume that this is also true for a pulse, the attenuation can be calculated.

Denoting the peak to peak amplitude of the primary echo, $A_{p}$, and the secondary echo, $A_{s}$, the attenuation can be calculated as

$$
\alpha=\frac{1}{2 d} \ln \left(\frac{A_{s}}{A_{p}}\right)
$$

The amplitudes $A_{p}$ and $A_{s}$ was measured from the sampled and digitized signals after they was scaled according to the gain and volt scale used.

Another way of estimate the attenuation [11] is to compare 
the energy, $E$, of the two echoes

$$
\frac{E_{s}}{E_{p}}=\frac{\int\left|y_{s}(t)\right|^{2} d t}{\int\left|y_{p}(t)\right|^{2} d t}=\frac{\left|\overline{A_{s}}\right|^{2}}{\left|\overline{A_{p}}\right|^{2}}
$$

where $y_{p}$ and $y_{s}$ is the primary and secondary echo. The integrals was calculated with Simpson's rule (see for example [10] p. 568) for each pulse. The attenuation is then found from

$$
\alpha=\frac{1}{2} \frac{1}{2 d} \ln \left(\frac{\left|\overline{A_{s}}\right|^{2}}{\left|\overline{A_{p}}\right|^{2}}\right)
$$

This gives a good estimation of the attenuation of the signals but it is sensitive to noise.

If the attenuation is frequency dependent, one must take into consideration that the attenuation of a pulse differs from the attenuation of a single frequency. To estimate the attenuation, only for the central frequency, a spectral method was used. The signals was Fourier transformed and the attenuation for each frequency, $\omega_{k}$, was calculated[12]. The discrete Fourier transform [10] was used

$$
W[k]=\frac{1}{N} \sum_{n=1}^{N} y[n] e^{-j 2 \pi(k-1)(n-1) / N}
$$

where $y[n]$ is the sampled signal of $y(t)$. A power spectrum of the pulses can be calculate as P.S. $=20 \log (|W[k]|)$ in terms of $d B$. The attenuation is found from

$$
\alpha[k]=\frac{1}{2 d} \ln \left(\frac{\left|W_{s}[k]\right|}{\left|W_{p}[k]\right|}\right)
$$

for each frequency.

The speed of the pulses was calculated by comparing the position of the maximum amplitude in time of the two echoes and by using cross-correlation.

The cross-correlation, $\Gamma$, is given by

$$
\Gamma[m]=\sum_{\langle n\rangle} y_{p}[n] y_{s}[n-m] \quad, \quad-N<m<N-1
$$

where the summation was taken for all points $n$ of the sampled pulses. Correlation gives a better estimation of the speed, especially for noisy signals, but also for pulses sampled at a low rate.

\section{RESUlts}

This section describes the measurements for each gas investigated. The experimental setup described above worked well for Ethane and Carbon Monoxide.

Measurements was also performed in Methane and Carbon Dioxide. However, no data could be sampled due to the high attenuation in these gases. The pulses vanished before they passed the measurement cell. [13].

\section{A. Ethane}

The measurements in Ethane worked very well. The attenuation is reasonably low for the measurement setup to work. The pressure was varied from $2 b a r$ to $8.4 b a r$ in steps of $0.2 b a r$. At lower pressure the signal was drowned by the noise.

The physical properties of Ethane at $300 \mathrm{~K}$ and 1 bar is listed below.

Physical Data of Ethane

\begin{tabular}{llll}
\hline \hline Density & $\rho_{0}$ & 1203 & $\mathrm{~g} / \mathrm{m}^{3}$ \\
Molar Weight & $M$ & 30.08 & $\mathrm{~g} / \mathrm{mol}$ \\
Specific Heat & $C_{P}$ & 53.1 & $\mathrm{~J} / \mathrm{mol} \mathrm{K}$ \\
Specific Heat & $C_{V}$ & 44.5 & $\mathrm{~J} / \mathrm{mol} \mathrm{K}$ \\
Ratio of Spf. H. & $\gamma$ & 1.193 & - \\
Viscosity & $\eta$ & 9.5 & $\mu \mathrm{Pas}$ \\
Thermal Conductivity & $\kappa$ & 21.3 & $\mathrm{~mW} / \mathrm{mK}$ \\
Speed of Sound & $c$ & 314.52 & $\mathrm{~m} / \mathrm{s}$ \\
Attenuation (1MHz) & $\alpha_{c}$ & 47.45 & $\mathrm{neper} / \mathrm{m}$ \\
Acoustical Impedance & $Z$ & 378.43 & $\mathrm{~kg} \mathrm{~m}^{2} / \mathrm{s}$ \\
\hline
\end{tabular}

The result of the spectral analysis is given i Fig. (4) for various value of the static pressure.

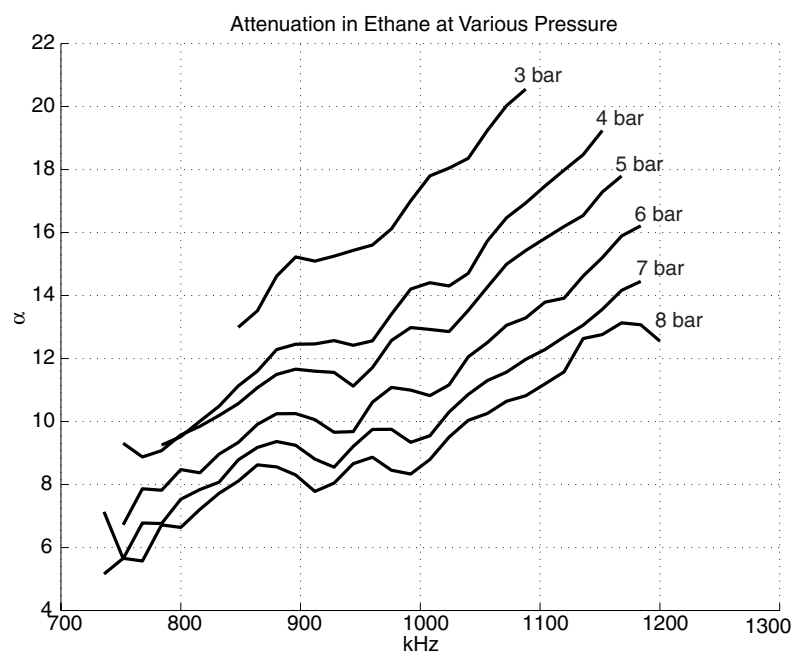

Fig. 4. The spectral attenuation in Ethane. Only points $20 d B$ above the noise floor was used in the calculations. This is the reason to the different length of the curves

The measured value of the attenuation is given in Fig. (5) and the speed of sound in Fig. (6) were the data have been scaled to frequency divided by pressure.

The variation of, especially the speed, but also the attenuation with frequency is noticeable. The speed varies from about $299 \mathrm{~m} / \mathrm{s}$ for low frequencies to $308 \mathrm{~m} / \mathrm{s}$ for high frequencies. A deduction is that the speed would reach $c_{\infty}=c_{0}$ for higher frequencies.

The lowest temperature recorded during the measurements was $21.7^{\circ} \mathrm{C}$ and the highest temperature recorded was $25.1^{\circ} \mathrm{C}$. This give rise to a great deal of uncertainty to the data. The deviation from the trend [1] in Fig. (6) is correlated to temperature fluctuations during the measurement.

The data is not sufficient to determine the relaxation time, $\tau$. 


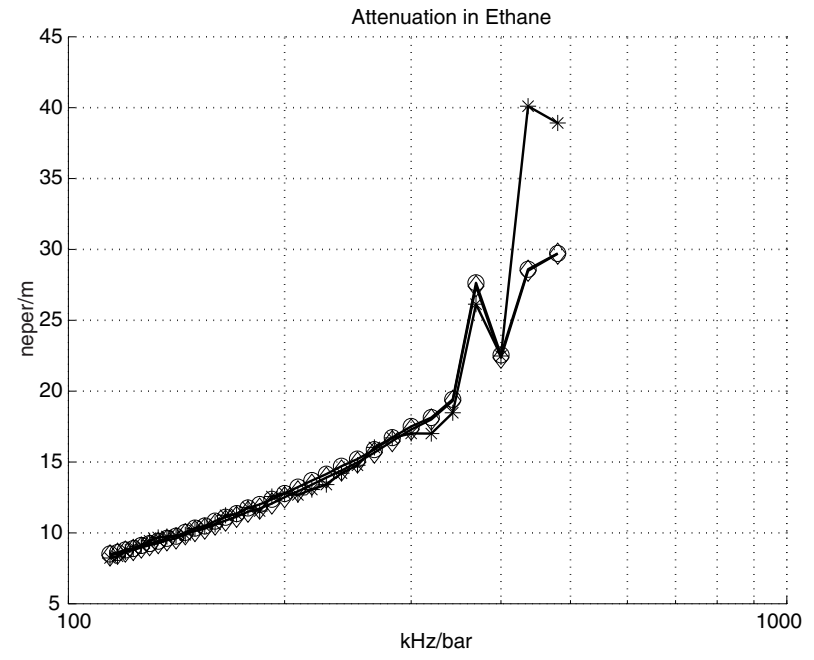

Fig. 5. Measured value of the attenuation in Ethene as function of frequency over pressure. ( ' $*$ '= at Center Frequency; 'o' = from amplitude; ' $\diamond '=$ from Energy)

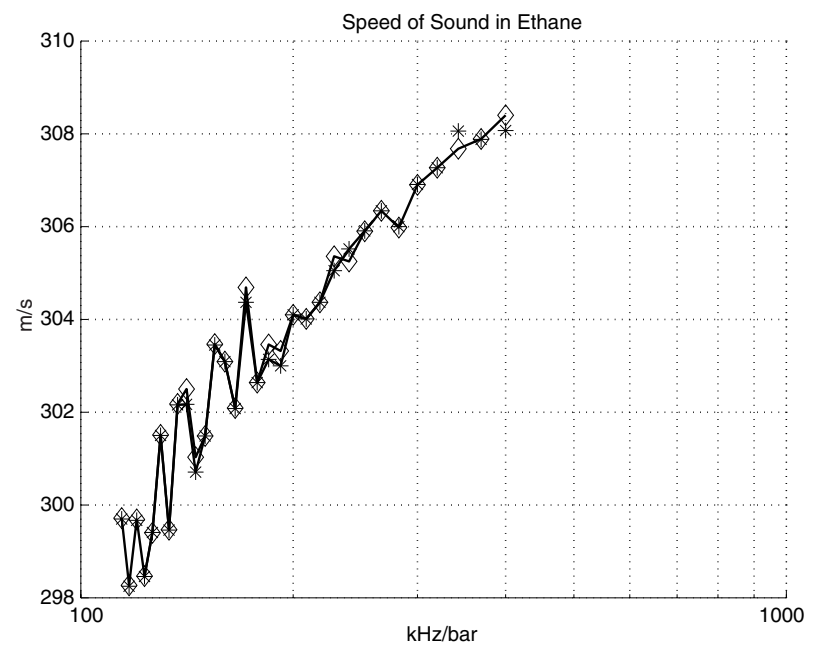

Fig. 6. The speed of sound in Ethane. (' $*$ ' $=$ estimated by comparing the time position of the maximum amplitudes; ' $\diamond$ ' = from cross correlation)

Since the speed of sound at high frequencies, $c_{\infty}$, nor the speed of sound at low frequencies, $c_{0}$, can be extracted.

On the other hand by comparing Fig. (6) to Fig. (1) we can see that the frequency region investigated probably do have a relaxation phenomena. However, it is impossible to determine wether the relaxation frequency, $\omega_{n}$, is within, or in the vicinity, of the frequency region.

Further measurements for both lower and higher frequencies is required, before any substantial conclusions can be made.

\section{B. Carbon Monoxide}

The measurements in Carbon Monoxide was performed without any trouble. The pressure was varied from 1bar to 9bar in steps of 0.2bar. The physical properties of Carbon Monoxide at $300 K$ and 1 bar is listed below.
Physical Data of Carbon Monoxide

\begin{tabular}{llll}
\hline \hline Density & $\rho_{0}$ & 1145 & $\mathrm{~g} / \mathrm{m}^{3}$ \\
Molar Weight & $M$ & 28.01 & $\mathrm{~g} / \mathrm{mol}$ \\
Specific Heat & $C_{P}$ & 29.14 & $\mathrm{~J} / \mathrm{mol} \mathrm{K}$ \\
Specific Heat & $C_{V}$ & 20.83 & $\mathrm{~J} / \mathrm{mol} \mathrm{K}$ \\
Ratio of Spf. H. & $\gamma$ & 1.399 & - \\
Viscosity & $\eta$ & 17.8 & $\mu \mathrm{Pas}$ \\
Thermal Conductivity & $\kappa$ & $25.0^{a}$ & $\mathrm{~mW} / \mathrm{mK}$ \\
Speed of Sound & $c$ & 353.98 & $\mathrm{~m} / \mathrm{s}$ \\
Attenuation & $\alpha_{c}$ & 143.33 & $\mathrm{neper} / \mathrm{m}$ \\
Acoustical Impedance & $Z$ & 404.16 & $\mathrm{~kg} \mathrm{~m}^{2} / \mathrm{s}$ \\
\hline a) Measured for $\rho \rightarrow 0$ & & &
\end{tabular}

The measured value of the attenuation is plotted in Fig. (7). The measurement of the attenuation is considerable lower than what was found from classical calculations. The reason for this, is probably, that the value of $\kappa$ is measured for a low pressure. Normally the thermal conductivity differs less than $1 \%$ between high and low pressures([13] p 6-185). However, in the case of Carbon Monoxide it seems that the listed value of $\kappa$ is unreliable. This explains why the value of the calculated attenuation, $\alpha_{c}$ is extremely high. A value of about a tenth of $\kappa$ gives good agrement for high frequencies.

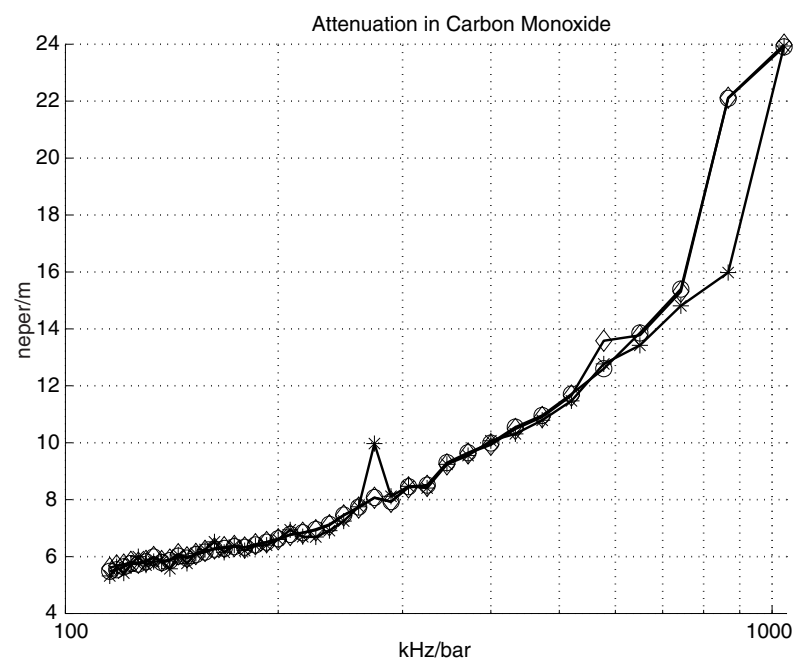

Fig. 7. Measured value of the attenuation Carbon Monoxide as function of frequency over pressure. ( ' $*$ ' $=$ at Center Frequency; ' $\circ$ ' = from amplitude; $' \diamond '=$ from Energy)

The speed of sound is plotted in Fig. (8). It is more or less constant. The measured speed is slightly less than expected from classical calculations. The reason to this is probably due to a bias error in the measurement of the distance between the transducer and the reflector. A constant speed that agrees reasonably well with classical calculations suggests that the relaxation phenomena occurs at lower frequencies than investigated.

The temperature varied from $19.46^{\circ} \mathrm{C}$ to $22.93^{\circ} \mathrm{C}$ during the measurement. The variation of the speed from the general trend in Fig. reffig-COSpeed is correlated to temperature fluctuations.

No conclusion about the relaxation time can be made from the measured data. A larger frequency scale has to be used. Further measurements are required. 


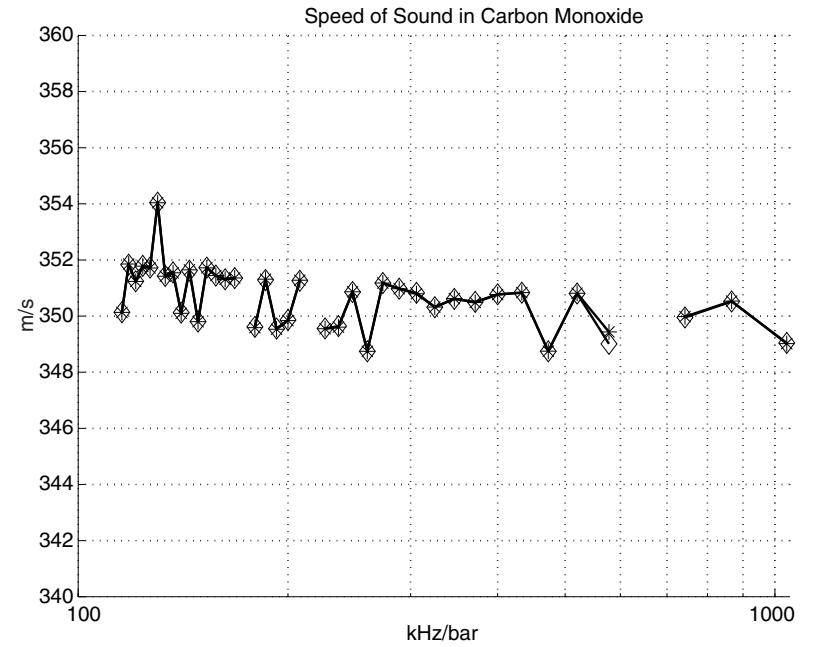

Fig. 8. The speed of sound in Carbon Monoxide. (' $*$ ' = estimated by comparing the time position of the maximum amplitudes; ' $\diamond$ ' = from cross correlation)

\section{DisCUSSION/CONCLUSIONS}

The major problem that occurred during the measurements was that the pulse generator used was not powerful enough to transmit pulses trough the gases. This can be overcome by the use of a more powerful transmitter. A higher efficiency can also be achieved by excite the transducer with pulses of a period time that is half the period time of the resonance frequency.

A larger frequency span have to be covered in order to determine the relaxation strength and the relaxation time as described above. This can be done by using an extremely broadband transducer or by using several narrow band transducers in order to cover a large frequency to pressure region. Also lower static pressures, near vacuum, can be used together with a high power pulser in order to reach high up on the frequency to pressure scale.

The pressure transmitter used measures the differential pressure compared to atmospheric pressure. The atmospheric pressure is typically 1 bar, but natural fluctuations is always present. Typically there is a high pressure during sunny days and a lower pressure during rainy days. By measure the atmospheric pressure, with a barometer, a better estimation of the pressure inside of the chamber can be achieved. A faulty measurement of the pressure gives an error on the frequency axis since the frequency is divided by pressure.

The temperature variation during the measurements is beyond an acceptable level. The reason for the temperature change is that a measurement currently takes several days to perform. For example, in the case of carbon monoxide, the measurement started on a Thursday and ended on Monday the week after. During a period of several days it is impossible to keep the temperature constant unless it is controlled. However, no facilities to control the temperature was available. The variation from the general trend of the speed in Fig. (6) and Fig. (8) is due to disparities of the temperature during the measurements.

No particular differences between the three algorithms used to determine the attenuation could be noticed except for low pressures. The same is valid for the two methods used to calculate the speed of sound. At low pressure, especially the secondary echoes recorded was very noisy. The signal to noise floor, at low pressure, for the secondary echoes were typically $25 \mathrm{~dB}$ to $30 d B$ for signal averaged 100 times.

Unfortunately no measured values of the relaxation times could be reported, which was the aim of the work. However, by study the shape of the celerity curve it can be concluded that Ethane probably have a relaxation frequency in the vicinity of the frequency to pressure region investigated.

The measured data of Carbon Monoxide is ambiguous, and no conclusions can be made without more experimental data available.

The acoustics of gases is an interesting problem. The next step in order to understand the acoustics of gases is to investigate the phenomena of sound velocity and absorption on a theoretically basis. There are little work published in the literature about the temperature dependency of the relaxation times. Also when it comes to gas mixtures one must survey which energy interactions that contribute to the relaxation phenomena.

\section{ACKNOWLEDGMENT}

The author wishes to send his gratitude to Mr. Jerry Lindblom of the EIS-Laboratories for his helpful comments and meaningful remarks during the design and construction of the measurement equipment. It would have been impossible without him.

Many thanks to Dr. Torbjörn Löfquist and Dr. Johan Carlson of the EIS-Laboratories for guiding me through the process of measure and analyze acoustic data.

Finally an appreciation to my fellow doctoral student Mr. Jonny Johanson for the many discussions we had during the execution of this project.

\section{REFERENCES}

[1] Delsing J., Blom I.,"On-line Masurement of Energy Content of Bio and Natural Gas Mixtures", in Swedish, Tech. Rep., ISSN 0282-3772,1995

[2] Attari A., Klass D. L.; "Natural Gas Energy Measurements", Elsevier Applied Science, 1987

[3] Report of IGU Subcommity B3, "Calculation of Properties and Predicted Behavior of Gases Based on Analysis", World Gas Conference, Milan, Italy, 1994

[4] Kinsler E. L. and Frey A. R., "Fundamentals of Acoustics", Wiley and Sons, 1982

[5] Dain Y., Lueptow R. M.,"Acoustic Attenuation in Three-Component Gas Mixtures - Theory", J. Acoust. Soc. Am., Vol. 109, p1955-1964, 2001

[6] Dain Y., Lueptow R. M.,"Acoustic Attenuation in Three-Component Gas Mixtures - Result", J. Acoust. Soc. Am., Vol. 110, p2974-2979, 2001

[7] Tanczos F. I., "Calculation of Vibrational Relaxation Times in the Chloromethanes", J. Chem. Phys., Vol. 22, p439-447, 1956

[8] Bhatia A. B., "Ultrasonic Absorption - An Introduction to the Theory of Sound Absorption and Dispersion in Gases, Liquids and Solids", Dover Publications, 1985

[9] Atkins P. W., "Physical Chemistry", Oxford University Press, (Fifth edition), 1994

[10] Harris J. W., Stocker H., "Handbook of Mathematics and Computational Science", Springer Verlag, 1998

[11] Carlson J., Martinsson P. E., "A Simple Scattering Model for Measuring Particle Mass Fractions in Multiphase Flow", Ultrasonics, No. 39, p585590, 2002

[12] Carlson J. "Ultrasonic Characterization of Materials and Multiphase Flow", doctoral thesis, ISSN: 1402-1544, 2002

[13] Lide D. R. (Editor in Chief), "Handbook of Chemistry and Physics", CRC Press LCC, 2000 